\title{
IL-27 targets Foxp3+ Tregs to mediate antiinflammatory functions during experimental allergic airway inflammation
}

\author{
Quang Tam Nguyen, Eunjung Jang, Hongnga T. Le, Sohee Kim, Dongkyun Kim, Nina Dvorina, \\ Mark A. Aronica, William M. Baldwin III, Kewal Asosingh, Suzy Comhair, and Booki Min
}

Department of Inflammation and Immunity, Lerner Research Institute, Cleveland Clinic Foundation, Cleveland, Ohio, USA

Foxp3 $3^{+}$CD4 Tregs are central regulators of inflammation, including allergic inflammation in the lung. There is increasing evidence that inflammatory factors undermine adequate Treg functions and homeostasis, resulting in prolonged and exacerbated inflammation. Therefore, identifying the factors is of the utmost important. IL-27 is an antiinflammatory cytokine implicated in immune regulation and tolerance. However, the cellular mechanisms underlying IL-27-mediated immune regulation in vivo remain largely unknown. Utilizing a cockroach antigen-induced allergic inflammation model in mice, we sought to test the roles of Tregs during IL-27-mediated regulation of allergic inflammation. Intranasally delivered IL-27 significantly reduced the development of airway inflammation. Unexpectedly, the IL-27-induced reduction occurred only in the presence of Tregs. II27 $\mathrm{ra}^{-/-}$and Treg-specific II27 $\mathrm{ra}^{-/-}$mice developed severe airway inflammation, and IL-27 treatment had little impact on diminishing the inflammatory responses. IL-27-induced treatment was restored following transfer of WT Tregs but not of Tregs deficient in Lag3, a molecule induced by IL-27 in Tregs. Finally, Tregs from asthmatic patients exhibited blunted STAT1 phosphorylation following IL-27 stimulation. Taken together, our results uncover that Tregs are the primary target cells of IL-27 in vivo to mediate its antiinflammatory functions, suggesting that altered IL-27 responsiveness in Tregs may underlie inadequate Treg functions and perpetuation of inflammation.

Conflict of interest: The authors have declared that no conflict of interest exists.

License: Copyright 2019, American Society for Clinical Investigation.

Submitted: June 27, 2018

Accepted: December 5, 2018

Published: January 24, 2019

Reference information: JCI Insight. 2019;4(2):e123216. https://doi.org/10.1172/jici. insight.123216.

\section{Introduction}

Asthma is a chronic inflammatory disease of the lung characterized by airway inflammation, airway hyperresponsiveness (AHR), and airway remodeling. In susceptible individuals, aberrant Th2-type immunity to otherwise harmless environmental antigens is considered a key factor triggering the pathogenesis of asthma through the secretion of Th2-type inflammatory cytokines, including IL-4, IL-5, and IL-13 (1). However, a cellular mechanism underlying the asthmatic inflammation is not fully understood.

Foxp3-expressing Tregs are central regulators of immunity and tolerance $(2,3)$. Defects in Treg generation and/or function are closely associated with uncontrolled lymphoproliferative diseases (4). Patients with the Foxp3 gene mutation exhibit allergic pathologies $(5,6)$, suggesting that Tregs are important in Th2-mediated allergic inflammation. Consistent with this, depleting Tregs prior to allergen challenge results in increased IgE production and severe inflammation (7). Likewise, adoptive transfer of allergen-specific Tregs prevents the development of AHR and airway inflammation (8). There is growing evidence that Treg homeostasis and functions can be impaired during allergic inflammation. For example, circulating CD25 Tregs in house dust mite-allergic children exhibit reduced suppressor function compared with nonasthmatic controls (9). In bronchoalveolar lavage fluid (BALF) from asthmatic patients, the proportion of Tregs is lower than that from healthy subjects, resulting in altered Treg/effector $\mathrm{T}$ cell ratio (10). Therefore, identifying a mediator that restores Treg homeostasis or enhances function may offer novel therapeutic approaches.

IL-27 is an IL-12 family cytokine composed of the p28 and Ebi3 subunits and produced by activated antigen presenting cells (APCs) $(11,12)$. IL-27 inhibits Th2 cell differentiation both in vitro and in vivo and Th2-type cytokine production from already-polarized Th2 effector cells (13-15). Concordantly, augmented allergic inflammation is observed in mice deficient in IL-27 receptor $\alpha$ (IL-27R $\alpha$ ), an IL-27-specific receptor subunit expressed on multiple cell types (16). While these studies have mainly focused on the impact of IL-27 on conventional T cells, the roles of other IL-27R $\alpha^{+}$cells such as Foxp $3^{+}$Tregs, which express higher 
level of the receptor during IL-27-mediated immune regulation, have mostly been overlooked. Mice deficient in IL-27 or IL-27R $\alpha$ have normal Treg development $(14,17)$, suggesting that IL-27 plays little role in Treg homeostasis under steady state conditions. However, IL-27 promotes T-bet and CXCR3 expression in Tregs that are specialized in controlling Th1-mediated immunity at local sites of inflammation (18), raising the possibility that IL-27 may play an important role in regulating Treg functions, especially during inflammation. We recently reported that IL-27 signaling in Foxp3 ${ }^{+}$Tregs is necessary to control Th17-mediated autoimmune inflammation (19). However, whether the IL-27/Treg axis is important in regulating Th2 associated allergic inflammation has not formally been tested.

In this study, utilizing a murine model of cockroach antigen-induced (CA-induced) airway inflammation (20), we sought to test the role of Tregs during IL-27-induced immune regulation. Intranasal IL-27 administration at allergen challenge significantly attenuated the development of allergic inflammation in the lung. To our surprise, the IL-27-induced inhibition was completely abolished in the absence of Tregs, suggesting that Tregs may be the key target cells of IL-27 in vivo. Indeed, adoptive Treg transfer into endogenous Treg-depleted mice fully restored IL-27-mediated inhibition of allergic inflammation. IL-27 induces the lymphocyte activation gene 3 (Lag3) in Tregs (19), and Lag3 expression in Tregs was required for IL-27 to inhibit allergic inflammation. The findings that Treg-specific $I l 27 \mathrm{ra}^{-1-}$ mice develop severe allergic inflammation that is not attenuated by systemic IL-27 administration further support the key contribution of IL-27 in Treg functions. Lastly, Tregs isolated from asthmatic patients displayed blunted IL-27induced STAT1 phosphorylation compared with those from control subjects, suggesting that such dampened responses may underlie altered Treg functions and further asthmatic inflammation. Taken together, these results highlight a nonreplaceable contribution of Tregs during IL-27-mediated regulation of allergic inflammation in the lung.

\section{Results}

Intranasal IL-27 administration attenuates CA-induced allergic airway inflammation. To test the potential of IL-27 in ameliorating experimental allergic inflammation, CA-mediated airway inflammation was induced, as shown in Figure 1A. Mice were sensitized with CA in alum adjuvant and intranasally challenged with CA in the presence or absence of IL-27. Cellular infiltration was determined 24 hours after the last challenge. Approximately $>90 \%$ of infiltrating cells in the BAL were eosinophils (Figure 1B). The absolute number of eosinophils in the BAL was significantly reduced in IL-27-treated mice compared with PBS-treated controls, while the numbers of neutrophils and CD4 T cells remained unchanged between the groups (Figure 1B). IL-27 treatment substantially diminished the number of lung infiltrating CD4 T cells and the accumulation of T cells expressing Th2-type (IL-4, IL-5, and IL-13), as well as other cytokines (IL-17, IFN- $\gamma$, IL-10, and IL-2), in the lung tissues compared with PBS-treated controls (Figure 1C). Interestingly, the IL-27-induced effects were only seen in the inflamed target tissues because CD4 T cell levels in the draining mediastinal lymph nodes (medLN) (Figure 1D) and in the nondraining lymph nodes (data not shown) remained comparable. Consistent with intracellular cytokine expression, IL-27 treatment diminished IL-4 secretion in the BALF (Figure 1E). IL-13 is the major driver of AHR in experimental allergic airway disease, acting independently of IL-4 on smooth muscle and epithelial cells $(21,22)$. Indeed, IL-13 secretion in the BALF was also reduced by the treatment (Figure 1E). Lung inflammation determined by H\&E staining and mucus production determined by PAS staining demonstrated that IL-27 treatment substantially diminished inflammation and mucus production (Figure $1 \mathrm{~F}$ ). Consistently, Muc5a and Muc5b mRNA expression in the lung tissues were dramatically reduced by the IL-27 treatment (Figure 1G). Lung functions (i.e., airway resistance) determined by flexivent experiment further supported the IL-27-mediated attenuation of the inflammation (Figure 1H). Therefore, IL-27 treatment reduces the hallmarks associated with allergic airway inflammation. Of note, the proportions of $\mathrm{T}$ cell subsets expressing inflammatory cytokines were similar between control and IL-27-treated mice (data not shown), suggesting that IL-27 blocks expansion and tissue infiltration of inflammatory cells rather than inhibits effector cell differentiation. Mice sensitized but not challenged with CA antigen did not develop any signs of inflammation in the lung (data not shown).

Intranasal IL-27 administration fails to inhibit airway inflammation without Foxp $3^{+}$Tregs, and adoptive Foxp $3^{+}$Treg transfer restores the inhibition. We recently reported that Foxp ${ }^{+}$Tregs control the development of allergic airway inflammation and that IL-27 signaling in Foxp3 ${ }^{+}$Tregs is essential to control Th17-mediated autoimmune inflammation $(19,20)$. Whether Tregs are essential during IL-27-mediated inhibition of allergic airway inflammation was next examined using Foxp $3^{\text {DTR }}$ mice (23). Tregs were depleted by diphtheria toxin (DTX) injection 


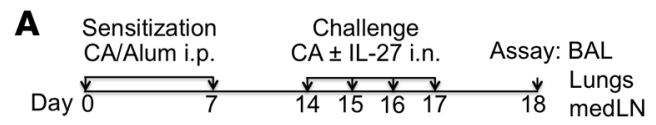

B
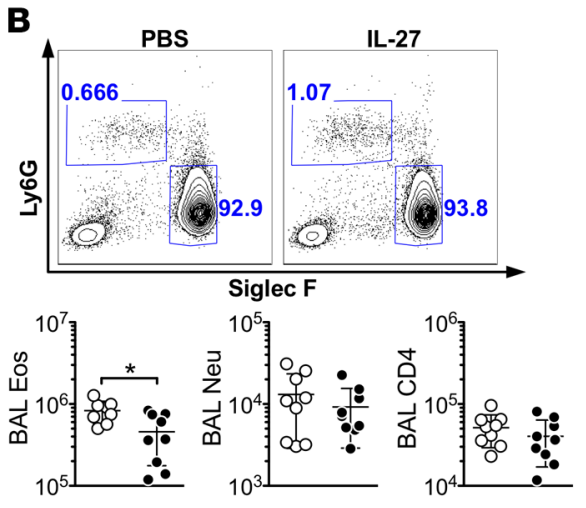

C
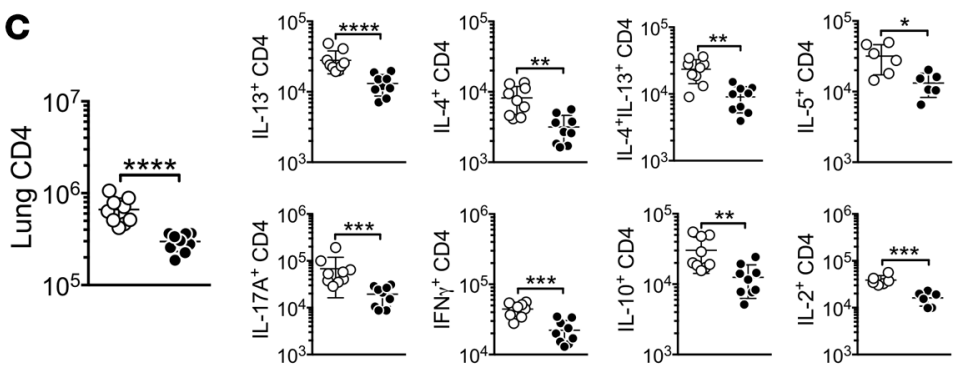

D

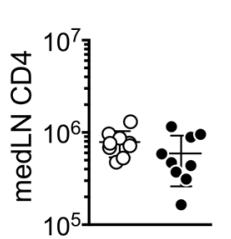

E

$\mathbf{F}$

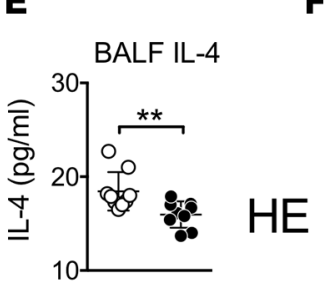

BALF IL-13

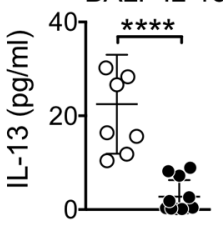

F

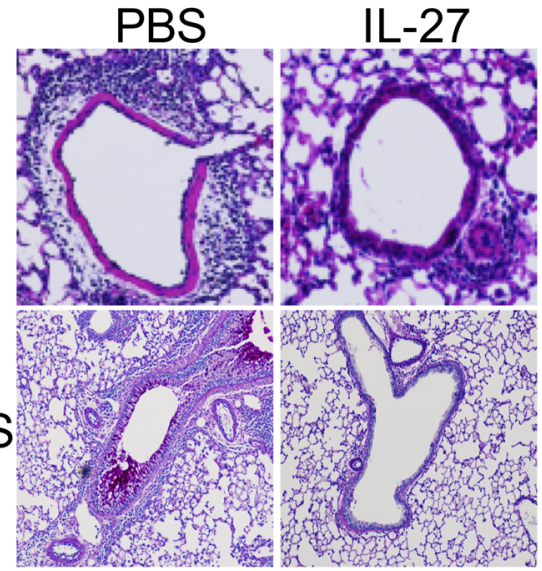

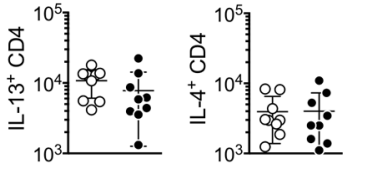
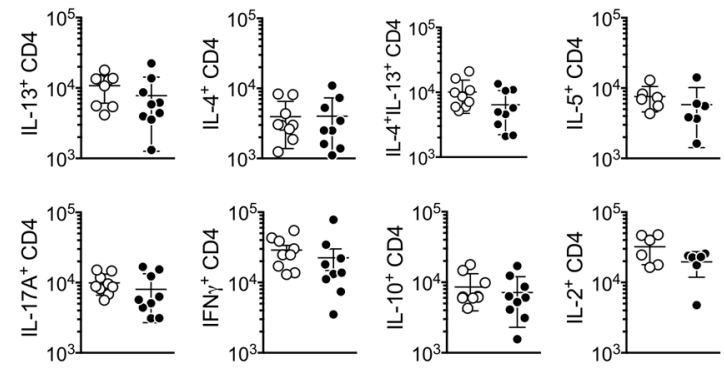

G
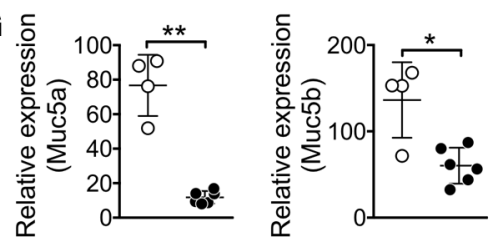

1

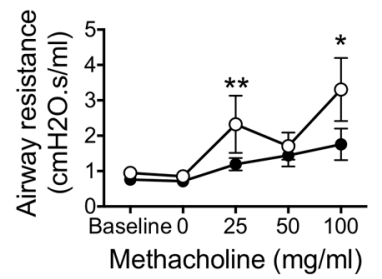

Figure 1. Intranasal IL-27 administration attenuates the development of allergic airway inflammation. (A) Experimental protocol. B6 mice were injected i.p. on days 0 and 7 with CA in alum adjuvant. Starting at day 14, mice were intranasally challenged for 4 consecutive days with CA alone or together with IL-27. Mice were sacrificed 24 hours after the last challenge. (B) BAL cells were examined for Ly6C and Siglec F expression. Differential cell count was performed by FACS analysis. (C and D) Lung (C) and draining medLN (D) cells were harvested and stimulated ex vivo to assess intracellular cytokine expression. (E) IL-4 and IL-13 secretion in the BALF was determined using a CBA assay. (F) H\&E and PAS staining of the lung tissues (original magnification $\times 20$ and $\times 10$, respectively) was used to evaluate inflammation. Histology score was determined as described in Methods. (G) Muc5a and Muc5b mRNA expression in the lung was determined by qPCR analysis. (H) Airway resistance was measured by flexivent experiments. Each symbol represents individually tested animal. The data shown are the mean \pm SD of 3 independent experiments $(n=9)$. ${ }^{*} P<$ $0.05,{ }^{* *} P<0.01,{ }^{* *} P<0.001,{ }^{* * *} P<0.0001$, as determined by Mann-Whitney nonparametric test. CA, cockroach antigen; BALF, bronchoalveolar lavage fluid; Eos, eosinophils; Neu, neutrophils; medLN, mediastinal lymph node.

1 day prior to and on the first day of CA challenge (Figure 2A). DTX-injected B6 WT mice were used as negative controls to ensure that any effects seen following DTX injection were not due to adjuvant effects of DTX. As previously reported (20), Treg depletion exacerbated allergic airway inflammation. The numbers of eosinophils and CD4 T cells infiltrating the airways were substantially elevated in Treg-depleted mice (Figure 2B). Lung-infiltrating CD4 T cells expressing inflammatory cytokines were elevated upon Treg deletion (Figure 2C). Interestingly, in the absence of Tregs, intranasal IL-27 treatment had no impact on downregulating inflammatory cell infiltration into the airways (Figure 2B) and the accumulation of cytokine-expressing effector CD4 $\mathrm{T}$ cells in the lung (Figure 2C). Similar Treg-dependent IL-27 treatment effects were also found in IL-4 and IL-13 secretion in the BALF (Figure 2D), as well as in lung inflammation (Figure 2E).

To ensure that the failure of IL-27-mediated inhibition in this condition is dependent on Tregs, in vitro generated Foxp $3^{+}$WT Tregs were adoptively transferred 1 day prior to Treg depletion (Figure 2A). IL-27-mediated treatment was restored when Tregs were transferred (Figure 2B). Inflammatory CD4 $\mathrm{T}$ cell accumulation in the lung was significantly reduced by IL-27 treatment when Tregs were 

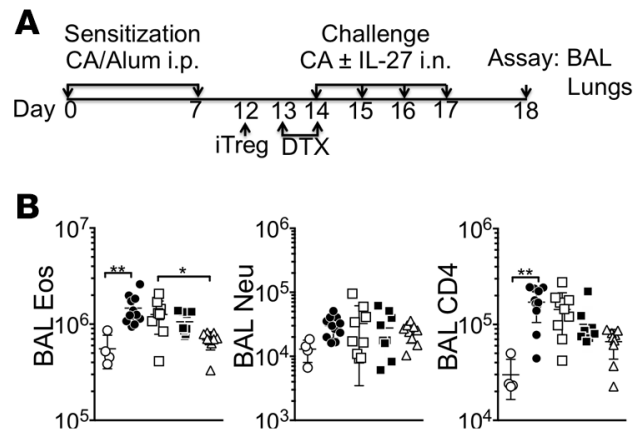

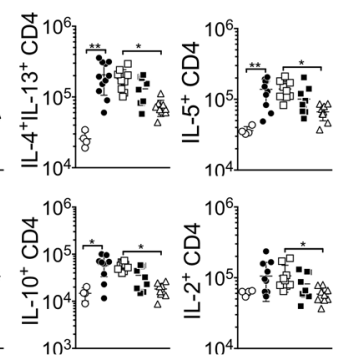

D
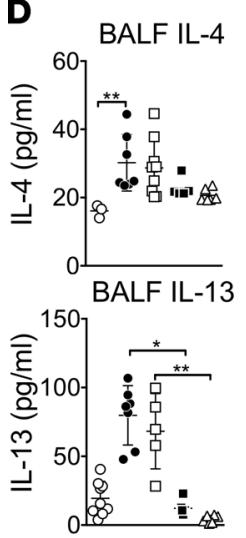

E $B 6 / D T X$

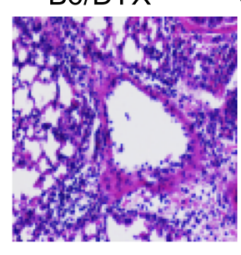

Foxp3.DTR/DTX/iTreg

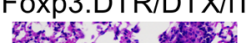

Foxp3.DTR/DTX Foxp3.DTR/DTX/IL-27
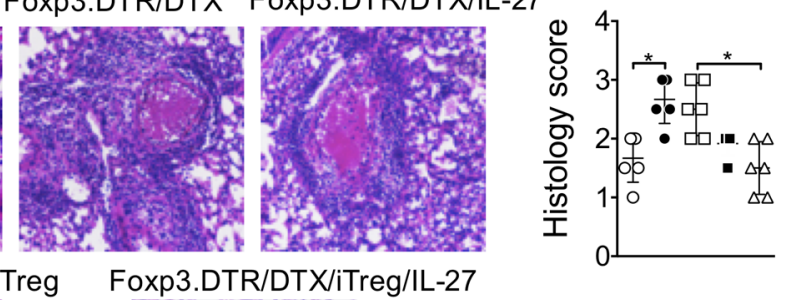

oxp3.DTR/DTX/iTreg/IL-27

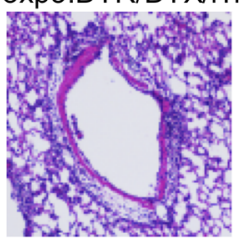

B B6/DTX

- Foxp3.DTR/DTX

$\square$ Foxp3.DTR/DTX/IL-27

- Foxp3.DTR/DTX/iTreg

$\triangle$ Foxp3.DTR/DTX/iTreg/L-27

Figure 2. Intranasal IL-27 administration fails to reduce airway inflammation in the absence of Foxp3+ Tregs, and adoptive Foxp3 ${ }^{+}$Treg transfer restores IL-27-mediated inhibition of airway inflammation. (A) Experimental protocol. B6 or B6 Foxp ${ }^{\text {DTR }}$ mice were sensitized with CA in alum adjuvant and intranasally challenged in the presence or absence of IL-27 as described in Figure 1. DTX was injected 1 day before and on the day of first Ag challenge. In some experiments, the mice received $2 \times 10^{6}$ in vitro-generated Foxp $3^{+}$Tregs 2 days before antigen challenge. Mice were sacrificed 24 hours after the last challenge. (B and C) Granulocytes and CD4 T cells in the BAL (B) and cytokine expressing CD4 T cells in the lung (C) were calculated by FACS analysis. (D) IL-4 and IL-13 secretion in the BALF was determined by a CBA assay. (E) H\&E staining of the lung tissues are shown (original magnification $\times 20$ ), and histology score was determined. All the experiments were repeated more than twice. The data shown indicate the mean \pm SD $(n=4-11)$. Each symbol represents an individually tested animal. ${ }^{*} P<0.05,{ }^{* *} P<0.01,{ }^{* *} P<0.001$, as determined by Kruskal-Wallis nonparametric test. CA, cockroach antigen; BALF, bronchoalveolar lavage fluid; Eos, eosinophils; Neu, neutrophils; iTreg, induced Tregs; DTX, diphtheria toxin.

transferred (Figure 2C). Likewise, both IL-4 and IL-13 secretion in the BALF, as well as lung inflammation, were dramatically reduced in these mice (Figure 2, D and E). Notably, transferring induced Tregs (iTregs) alone without IL-27 treatment was sufficient to reduce inflammatory CD4 T cell accumulation in the lung, cytokine secretion in the BALF, and lung inflammation, although IL-27 cotreatment further improved Treg-mediated inhibition (Figure 2, C-E). Therefore, these results demonstrate that Tregs are critical players during IL-27-mediated inhibition of allergic airway inflammation, raising the possibility that Foxp $3^{+}$Tregs may be the essential targets of IL-27 in vivo.

Foxp $3^{+}$Tregs are the direct targets for IL-27 to diminish allergic airway inflammation. IL-27R $\alpha$, the IL-27-specific subunit of the IL-27 receptor complexes, is expressed on multiple cell types, including lymphocytes and APCs (24). To investigate whether Tregs are the primary targets of IL-27 to control airway inflammation in vivo, IL-27R $\alpha$-deficient (Il27 $\left.\mathrm{ra}^{-/}\right)$mice were utilized. Compared with WT controls, $I l 27 \mathrm{ra}^{-1-}$ mice developed severe CA-induced lung inflammation. Infiltration of inflammatory cells in the BAL and accumulation of CD4 T cells expressing inflammatory cytokines in the lung were substantially elevated in $\mathrm{Il}_{2} 7 \mathrm{ra}^{-/-}$mice (Figure 3, A and B). Interestingly, the number of neutrophils in the BAL was also increased in these mice (Figure 3A). Histopathological examination further revealed substantial lung inflammation in $\mathrm{Il}_{27 \mathrm{ra}^{-1-} \text { mice }}$ (Figure 3C). As expected, intranasal IL-27 administration had no impact on downregulating inflammation (Figure 3, A-C). Transferring WT iTregs into $1727 \mathrm{ra}^{-/-}$mice alone was sufficient to downregulate inflammatory cell infiltration in the airway (Figure 3A), inflammatory CD4 $\mathrm{T}$ cell responses in the lung (Figure 3B), and lung inflammation (Figure 3C). IL-27 cotreatment in these conditions further improved IL-27-mediated inhibition of airway inflammation (Figure 3, A-C). Since transferred Tregs are the only IL-27-responding cells in this system, these results suggest that Foxp $3^{+}$Tregs alone can mediate inhibitory effects of IL- 27. 
A

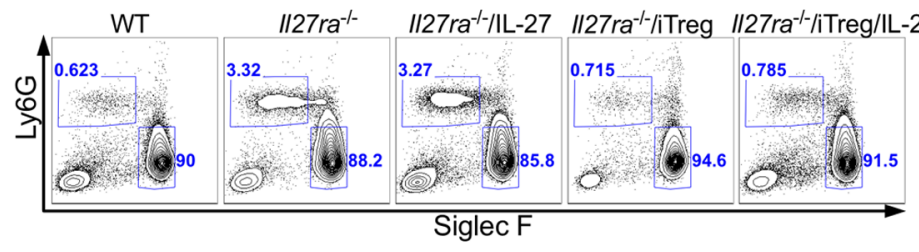

C

C WT

II27ra $\mathrm{ra}^{-1-}$

II27 $\mathrm{ra}^{-/-/ I L-27}$
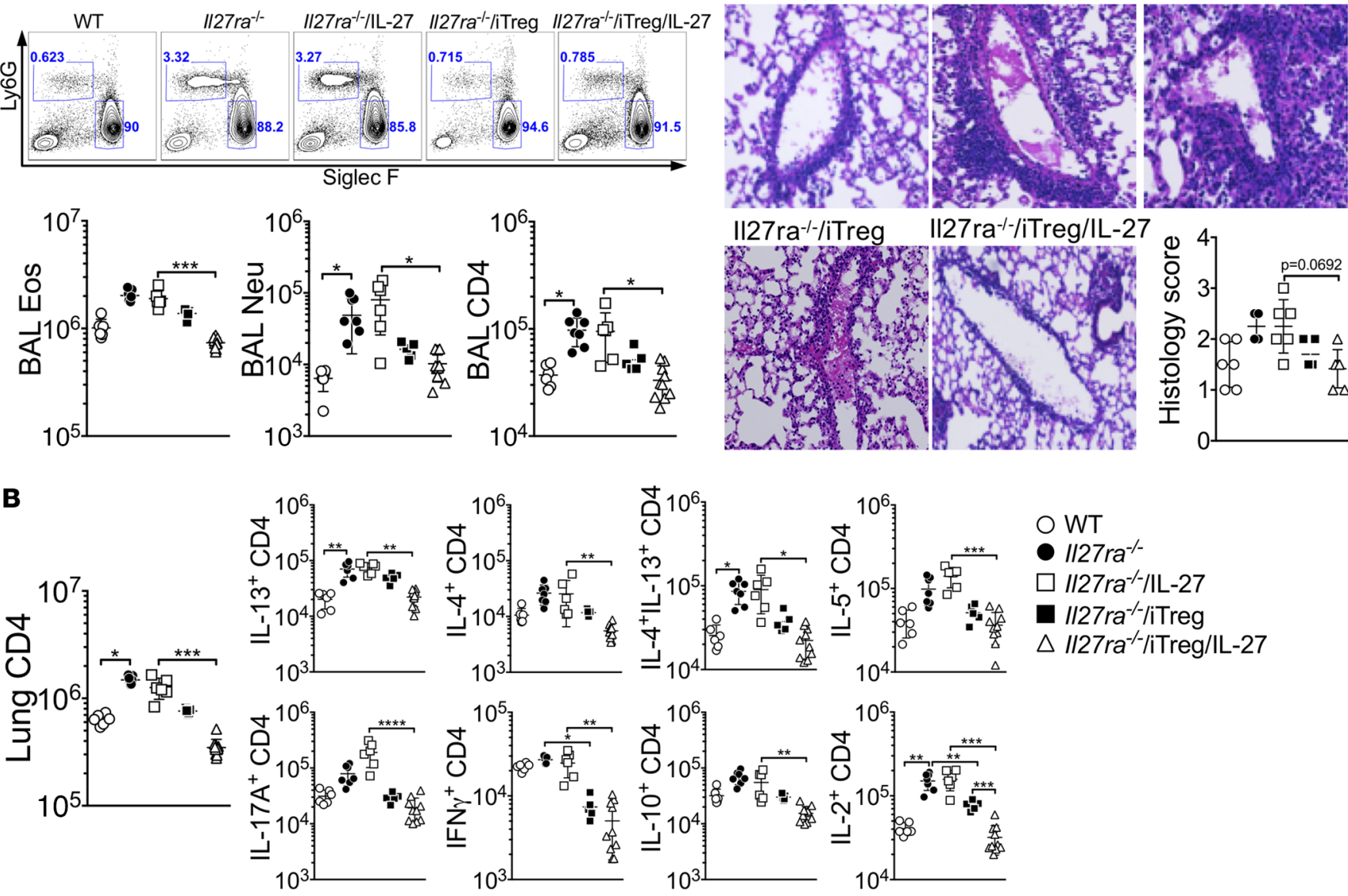

Figure 3. Transfer of WT Tregs into II27 $\mathrm{ra}^{-/-}$mice diminishes allergic airway inflammation after IL-27 treatment. WT and II27ra $/$mice were sensitized with CA in alum adjuvant and intranasally challenged in the presence or absence of intranasal IL-27. In some experiments, $2 \times 10^{6}$ iTregs were transferred 2 days before antigen challenge. Mice were sacrificed 24 hours after the last challenge. (A) BAL cells were examined for Ly6C and Siglec F expression, and differential cell counts was performed by FACS. (B) Lung cells were stimulated ex vivo, and intracellular cytokine expression was determined. (C) H\&E staining of the lung tissues are shown (original magnification $\times 20$ ), and histology score was evaluated. The data shown represent the mean \pm SD of 2 independent experiments $(n=6)$. Each symbol represents an individually tested mouse. ${ }^{*} P<0.05,{ }^{*} P<0.01,{ }^{* * *} P<0.001$, as determined by Kruskal-Wallis nonparametric test. BAL, bronchoalveolar lavage; Eos, eosinophils; Neu, neutrophils; iTreg, induced Tregs.

Allergen-induced allergic airway inflammation is exacerbated in Treg-specific Il27ra ${ }^{-1-}$ mice. To precisely determine the role of IL-27 signaling in Treg functions during allergic airway infiltration, Treg-specific Il27ra ${ }^{\prime-}$ mice (Treg ${ }^{\Delta I I 27 a}$ ) were next used (19). Treg ${ }^{\Delta I I 27 r a}$ and littermate Treg $^{\mathrm{WT}}$ control mice were induced for allergic airway inflammation. Infiltration of inflammatory cells in the BALF was significantly elevated in Treg ${ }^{\Delta I I 27 r a}$ mice compared with that in Treg ${ }^{\mathrm{WT}}$ mice (Figure 4A). Lung-infiltrating CD4 T cells expressing inflammatory cytokines were more abundant in Treg ${ }^{\Delta I I 27 r a}$ mice (Figure 4B). Lung tissues of Treg ${ }^{\Delta I / 27 r a}$ mice were heavily infiltrated with inflammatory cells (Figure 4C). Interestingly, Treg infiltration in the lung of Treg ${ }^{\Delta I I 7 r a}$ mice was also elevated, suggesting that IL-27 controls Treg functions but not Treg survival or trafficking (Figure 4D). IHC analysis further confirmed substantial Treg accumulation in the lung of Treg $^{\Delta I I 27 r a}$ mice (data not shown). To corroborate that IL-27 signaling in Tregs is instrumental in controlling inflammation, Treg ${ }^{\Delta I I 27 r a}$ and Treg ${ }^{\mathrm{WT}}$ mice induced for allergic airway infiltration received intranasal IL-27, as shown in Figure 1. IL-27 treatment significantly reduced the numbers of lung-infiltrating inflammatory CD4 T cells in TregWT but not in Treg ${ }^{\Delta I 27 r a}$ mice (Figure 4E). These results strongly suggest that IL-27 signaling in Foxp3 ${ }^{+}$Tregs is essential for Treg function to control allergic airway infiltration.

IL-27 inhibits effector $T$ cell proliferation via Tregs. One potential mechanism by which Tregs control inflammatory responses is to suppress effector $\mathrm{T}$ cell proliferation $(25,26)$. We thus investigated whether intranasal delivery of IL-27 suppresses T cell proliferation and whether Tregs are necessary during the process. In vivo proliferation of CD4 T cells was determined by BrdU incorporation. Foxp $3^{\mathrm{DTR}}$ mice induced for the inflammation were injected with BrdU 24 hours before sacrifice. Indeed, the percentage of BrdU ${ }^{+}$ 

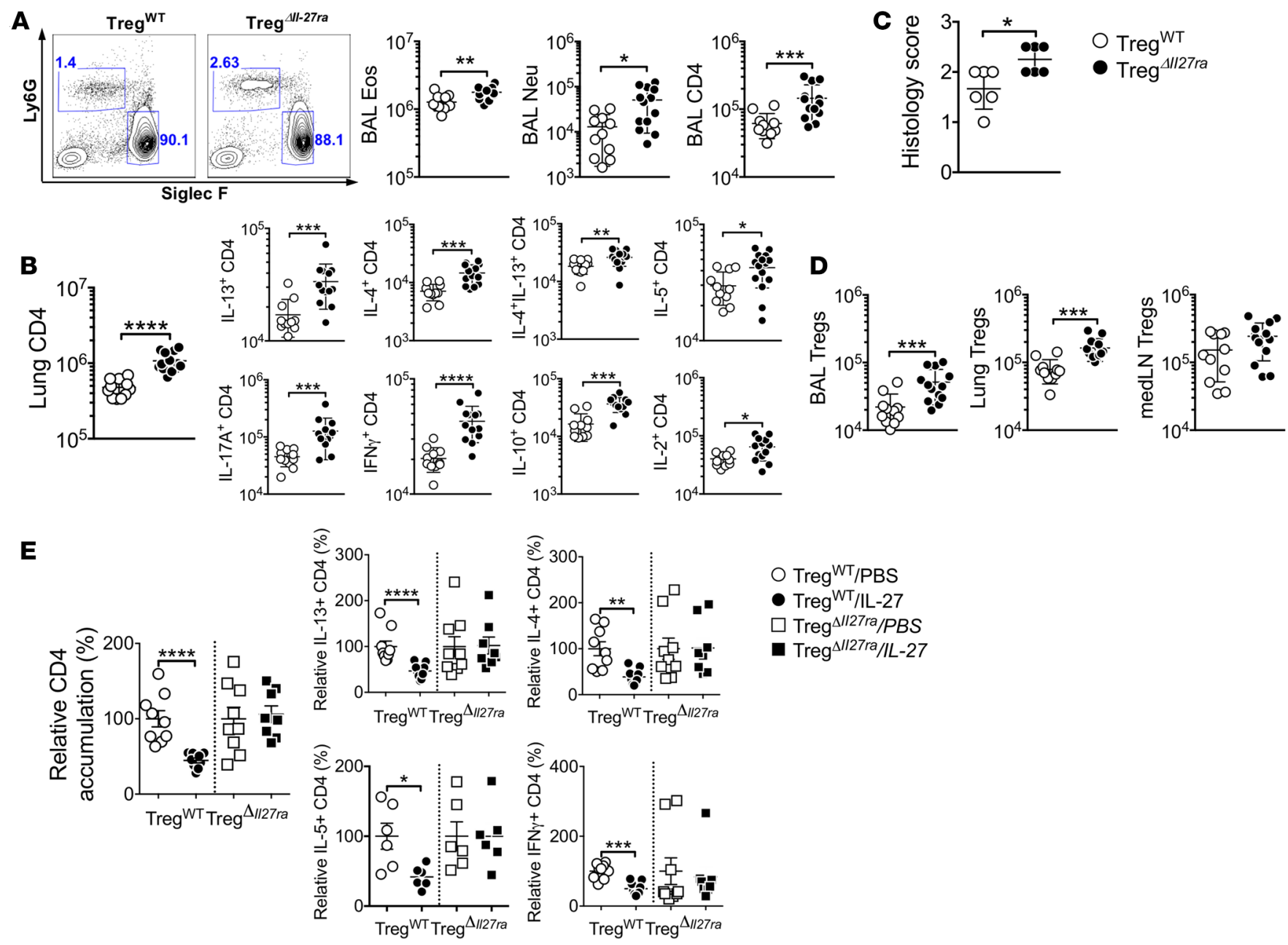

Figure 4. Treg-specific II27 $\mathrm{ra}^{-/-}$mice have exacerbated allergic airway inflammation. Treg-specific $1 / 27 \mathrm{ra}^{-1-}$ (Treg ${ }^{\Delta I 127 \mathrm{ra}}$ ) and littermate control mice were sensitized with CA in alum adjuvant and intranasally challenged as described above. (A) BAL cells were examined for Ly6G and Siglec F expression, and differential cell counts was performed by FACS. (B) Lung cells were stimulated ex vivo, and intracellular cytokine expression was determined. (C) Histology score was determined. (D) Foxp3 ${ }^{+}$Tregs were enumerated from the indicated tissues. (E) IL-27 was intranasally administered during antigen challenge as described in Figure 1. Relative CD4 accumulation in the lung, as well as cytokine expression, were compared. The data shown represent the mean \pm SD of more than 2 independent experiments $(n=11-13)$. Each symbol represents an individually tested mouse. ${ }^{*} P<0.05,{ }^{* *} P<0.01,{ }^{* * *} P<0.001,{ }^{* * * *} P<0.0001$ as determined by Mann-Whitney nonparametric test. BAL, bronchoalveolar lavage; Eos, eosinophils; Neu, neutrophils.

effector T cells in the lung was significantly reduced in IL-27-treated mice compared with PBS-treated controls (Figure 5A). Interestingly, Treg proliferation itself was also reduced by IL-27 treatment (Figure 5A). Upon Treg depletion, effector T cell proliferation was dramatically increased and IL-27 completely lost its inhibitory functions to interfere with effector $\mathrm{T}$ cell proliferation, as $\mathrm{BrdU}$ incorporation of effector $\mathrm{T}$ cells remained increased (Figure 5A). Surface expression of molecules implicated in Treg suppression, including ICOS, Nrp1, CD39, and CD73, remained unchanged by IL-27 treatment, suggesting that IL-27 regulates Treg functions by a mechanism independent of these molecules (Figure 5B). We recently reported that lung-infiltrating effector CD4 T cells express ICOS and Nrp1 and that the expression correlates with the extent of activation (20). Concordantly, their expression in effector cells was also reduced by IL-27, while dramatically increased by Treg depletion, regardless of IL-27 (Figure 5B). Tregs can exert suppressive function by inactivating APCs (27). However, we found that surface expression of costimulatory molecules such as CD80, CD86, and ICOS ligand on $\mathrm{CD} 11 \mathrm{c}^{+} \mathrm{CD} 11 \mathrm{~b}^{+}$and $\mathrm{CD} 11 \mathrm{c}^{-} \mathrm{CD} 11 \mathrm{~b}^{+}$cells in the lung remained unchanged by IL-27 stimulation (Supplemental Figure 1; supplemental material available online with this article; https://doi.org/10.1172/jci.insight.123216DS1).

In vivo BrdU incorporation experiment was repeated in $\mathrm{Il}_{2} 7 \mathrm{ra}^{-1-}$ mice. The proportion of proliferating CD4 T cells in the lung was comparable between WT and $\mathrm{Il}_{27 \mathrm{ra}^{-1-}}$ mice (Figure $5 \mathrm{C}$ ), despite 
A
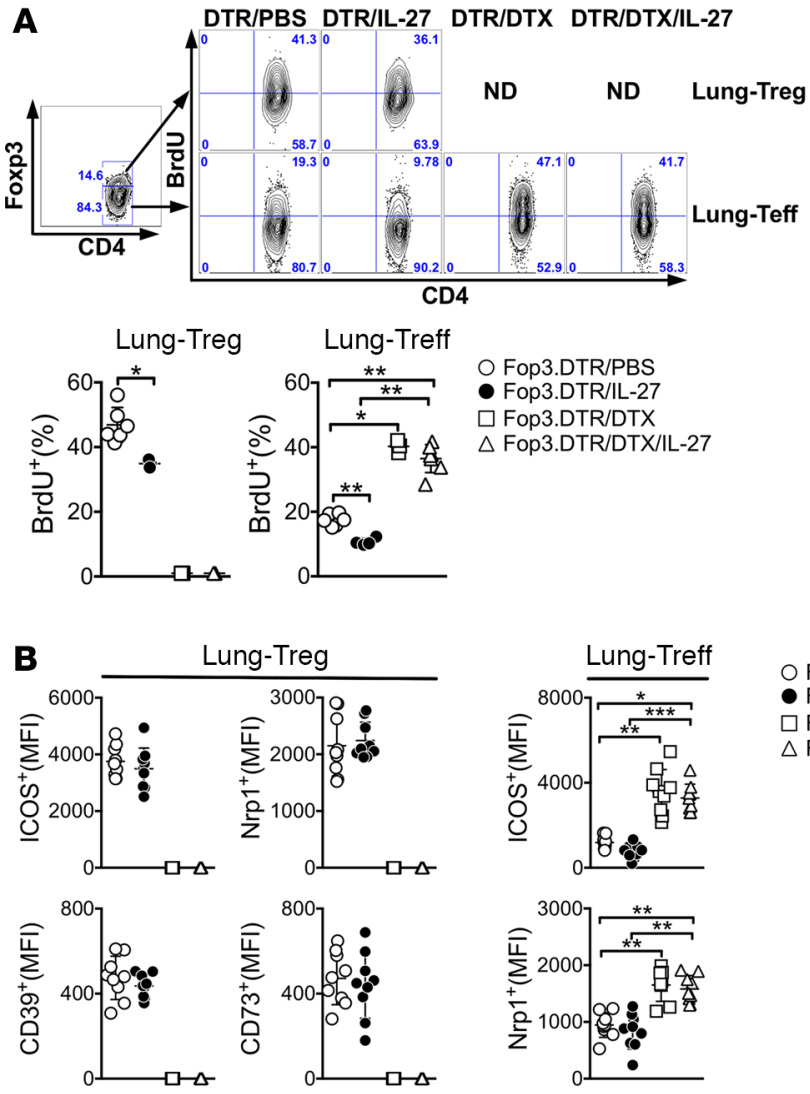

Lung-Treg
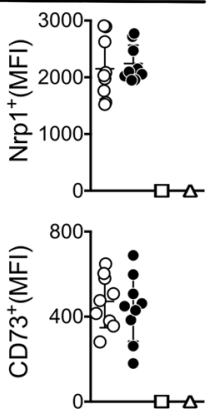

O Fop3.DTR/PBS

- Fop3.DTR/IL-27

$\square$ Fop3.DTR/DTX

$\triangle$ Fop3.DTR/DTX/IL-27
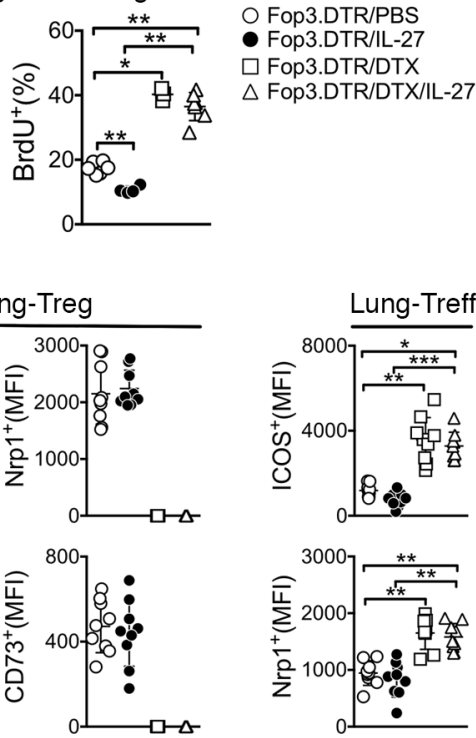
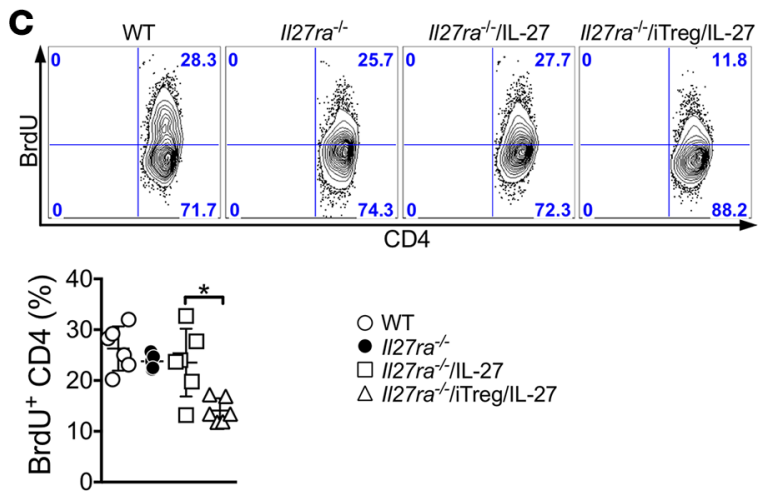

O WT

- $1127 \mathrm{ra}^{-/-}$

$\triangle 1 / 27 \mathrm{ra}^{-/}$-liTreg/LL-27

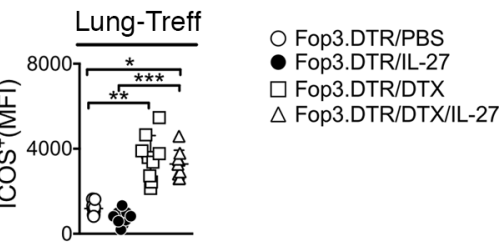

Figure 5. IL-27 inhibits effector T cell proliferation via Treg. (A) Foxp $3^{\text {DTR }}$ mice were sensitized with CA in alum adjuvant and intranasally challenged in the presence or absence of IL-27 as described above. DTX was also injected as indicated. BrdU was injected 24 hours prior to sacrifice. BrdU incorporation was determined by FACS analysis as described in Methods. (B) Treg and effector T cell expression of the listed surface molecules was determined by FACS analysis. (C) $1127 \mathrm{ra}^{-/-}$mice were treated as described above. BrdU incorporation was determined by FACS analysis as described. The data shown represent the mean \pm SD of 2 independent experiments $(n=4-9)$. Each symbol represents an individually tested mouse. ${ }^{*} P<0.05$, ${ }^{*} P<0.01,{ }^{* * *} P<0.001$, as determined by Mann Whitney (A and C) and by Kruskal-Wallis (B) nonparametric test. DTX, diphtheria toxin; iTreg, induced Treg.

the fact that $\mathrm{Il2}_{2} \mathrm{ra}^{-1-}$ mice developed more robust inflammatory responses (Figure 3, B and C). IL-27 administration did not affect $\mathrm{T}$ cell proliferation in $\mathrm{Il2}_{2} \mathrm{ra}^{-1-}$ mice (Figure 5C). However, adoptive transfer of WT iTregs was sufficient to downregulate CD4 T cell proliferation in these conditions (Figure 5C), strongly suggesting that IL-27 signaling in Tregs is necessary and sufficient in suppressing effector $\mathrm{T}$ cell proliferation.

Lag3 expression on Tregs is necessary for IL-27 to suppress inflammatory responses via Tregs. We previously reported that IL-27 induces Treg expression of Lag3, a CD4-like molecule implicated in Treg functions, and that Lag3 expression on Tregs plays a key role in mediating Treg function during intestinal inflammation (28). To test whether Lag3 expression on Tregs is necessary during IL-27-mediated inhibition of allergic airway inflammation, in vitro-generated $\operatorname{Lag} 3^{-1-}$ iTregs were transferred into Treg-depleted mice, followed by intranasal IL-27 treatment. While IL-27 administration significantly diminished inflamma-

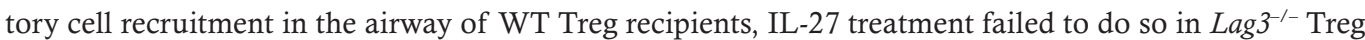
recipients (Figure 6A). Similarly, the accumulation of inflammatory CD4 T cells in the lung was substan-

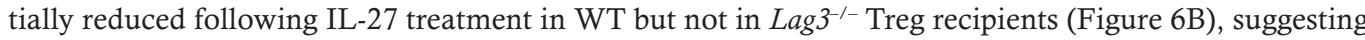
that Lag3 is indeed a mediator of IL-27 functions for Tregs to control allergic inflammation. In support, Lag3 expression on lung-infiltrating Foxp $3^{+}$Tregs was substantially lower in Treg ${ }^{4 I 27 r a}$ compared with that in Treg ${ }^{\mathrm{WT}}$ mice, whereas lung infiltrating effector CD4 T cells expressed comparable levels of Lag3 (Figure 6C). Treg-derived IL-10 is considered a key mediator of Treg function (29). However, we found that IL-10 expression both on lung-infiltrating Foxp $3^{+}$Tregs as well as effector CD4 T cells was comparable between Treg ${ }^{\Delta I 27 r a}$ and Treg ${ }^{\mathrm{WT}}$ mice (Figure 6C). Therefore, Lag3 expression on Tregs appears to be essential for IL-27 to control allergic inflammation via Tregs. 

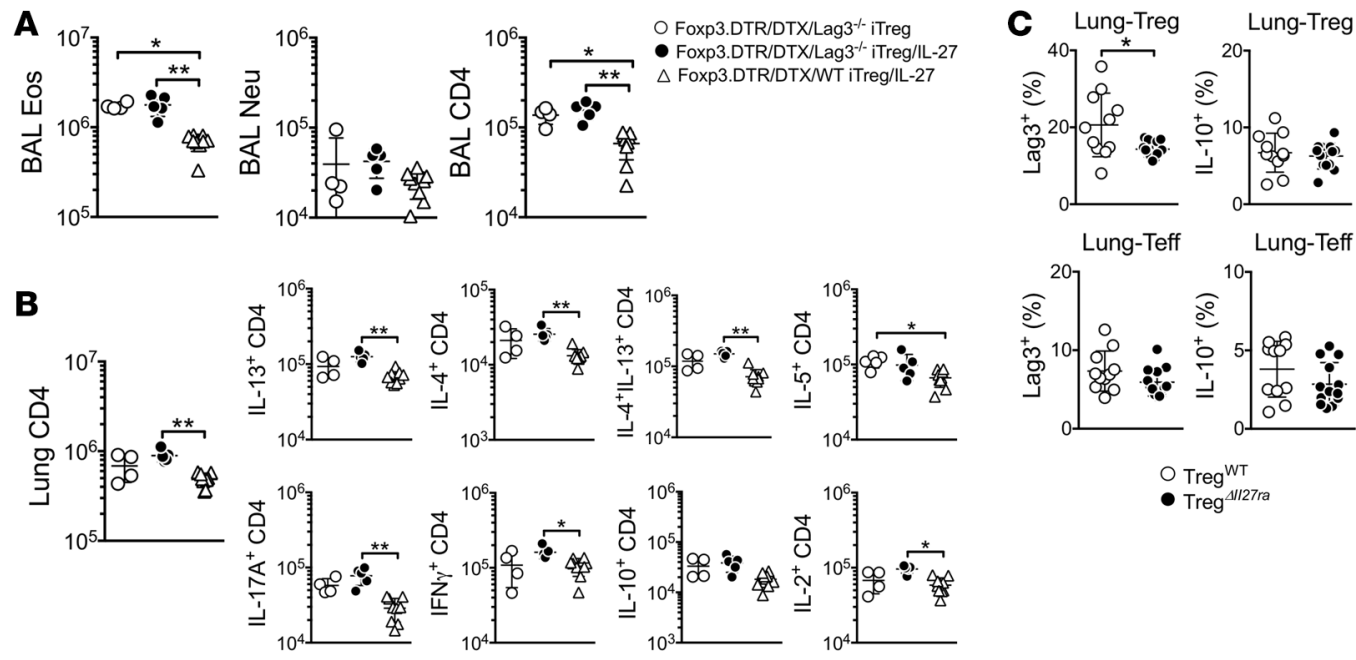

O Treg
- Treg
Trezra

Figure 6. Lag3 expression on Treg is necessary for Treg-mediated suppression in response to IL-27. (A and B) Foxp $3^{\text {DTR }}$ mice were sensitized with CA in alum and intranasally challenged in the presence or absence of IL-27 as described above. DTX was injected 1 day before and on the day of first Ag challenge. Then, the mice received $2 \times 10^{6}$ in vitrogenerated Foxp3 ${ }^{+}$Tregs deficient in Lag3 two days before antigen challenge. Mice were sacrificed 24 hours after the last challenge. Granulocytes and CD4 T cells in the BAL (A) and cytokine-expressing CD4 T cells in the lung (B) were calculated by FACS analysis. (C) Treg ${ }^{\mathrm{WT}}$ and Treg ${ }^{\Delta \| 127 r a}$ mice were sensitized with CA in alum and intranasally challenged as described above. Upon sacrifice, Lag3 and IL-10 expression of lung-infiltrating conventional CD4 and Foxp3+ Tregs was measured by FACS analysis. All the experiments were repeated more than twice. The data shown indicate the mean $\pm S D(n=4-13)$. Each symbol represents an individually tested animal. ${ }^{*} P<0.05,{ }^{*} P<0.01$, as determined by Kruskal-Wallis nonparametric test. BAL, bronchoalveolar lavage; Eos, eosinophils; Neu, neutrophils.

Blunted STAT1 phosphorylation in Tregs from asthmatic patients in response to ex vivo IL-27 stimulation. Based on the results that IL-27 stimulation of Tregs is essential for Treg functions to control inflammatory responses, we hypothesize that Tregs from asthmatic patients may exhibit intrinsically altered IL-27 responses, which may affect their adequate function. Since IL-27 delivers its activation signal through phosphorylation of both STAT1 and STAT3 (30-34), we compared IL-27-induced pSTAT1 and pSTAT3 expression ex vivo utilizing Tregs and conventional CD4 T cells isolated from asthmatic patients and control donors. Peripheral blood mononuclear cells (PBMCs) were incubated with or without IL-27 for the indicated durations. Phosphorylation of STAT1 and STAT3 in human Tregs $\left(\mathrm{CD} 4^{+} \mathrm{CD} 25^{+} \mathrm{CD} 127^{-}\right)$and conventional CD4 T cells $\left(\mathrm{CD}^{+} \mathrm{CD} 25^{-} \mathrm{CD} 127^{+}\right)$was determined by flow cytometry. IL-27-induced STAT1 phosphorylation in patient Tregs was significantly reduced compared with that in control donor Tregs (Figure 7A). STAT3 phosphorylation seemed slightly lower in patient Tregs, although the difference did not reach statistical significance. Interestingly, conventional CD4 T cells from asthmatic patients also exhibited similarly lower levels of STAT1 and STAT3 phosphorylation compared with control donors (Figure 7A). Different susceptibility to IL-27-induced STAT phosphorylation was not due to IL-27 receptor expression level, as the level of the receptor expression was comparable between the groups, regardless of $\mathrm{T}$ cell types (Figure 7B). Notably, the patient samples analyzed in this study were all from females (4 severe and 2 nonsevere asthmatics), while the control samples were from both sexes ( 2 females and 4 males). However, pSTAT1 upregulation in female patient Tregs was lower when compared with that in female or male controls (data not shown). We did not notice sex differences in pSTAT expression within control samples. Moreover, pSTAT expression was comparable between severe and nonsevere asthmatics. Taken together, these results demonstrate that Tregs from asthmatic patients exhibit intrinsic defects of STAT1 and STAT3 phosphorylation in response to IL-27 stimulation, possibly contributing to inadequate regulatory functions of Tregs.

\section{Discussion}

Regulatory functions of IL-27 during inflammatory responses have previously been proposed, since mice deficient in IL-27p28 or IL-27R $\alpha$ are highly susceptible to autoimmune and allergic inflammation (14, 3540). However, the precise in vivo target cells of IL-27 and underlying cellular mechanisms by which IL-27 mediates such functions remain largely unclear. We previously reported, using a $\mathrm{T}$ cell-mediated colitis 
A Treg
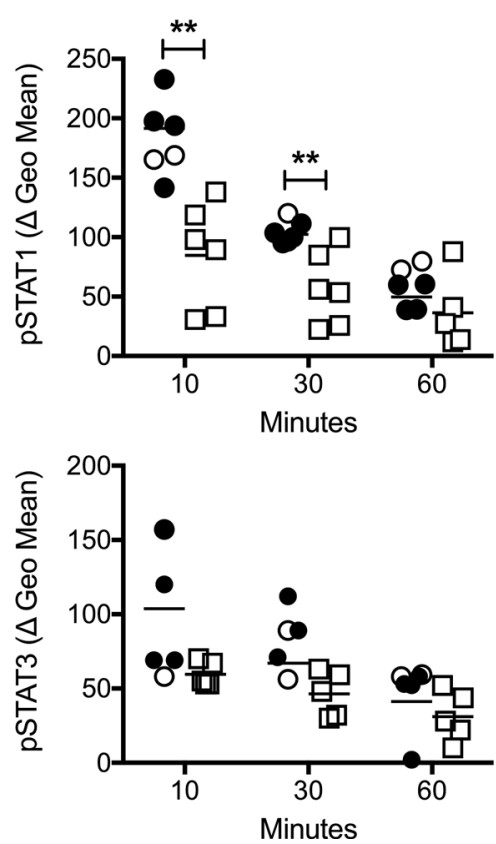

Tcon

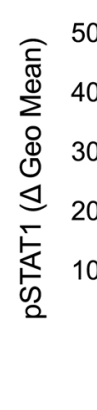

Female Healthy Donors

- Male Healthy Donors

$\square$ Female Patients

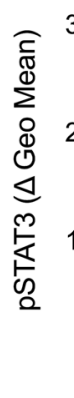

B
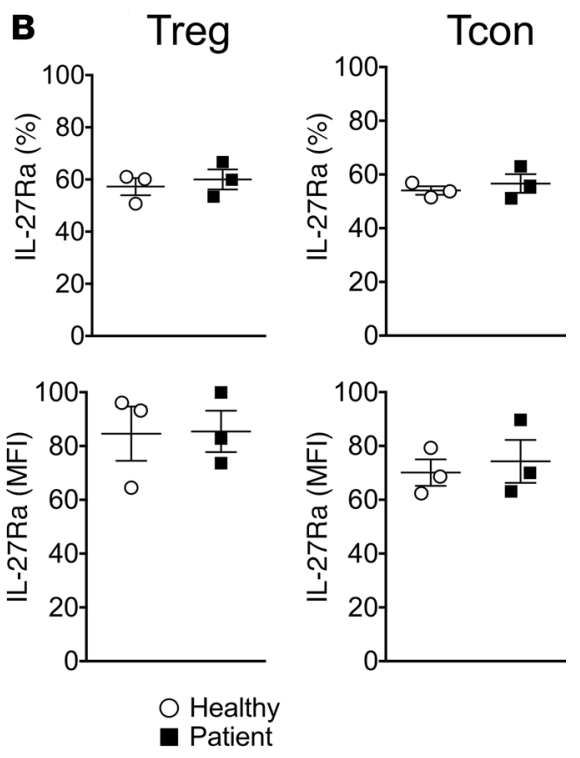

Figure 7. Phosphorylation of STAT1 in response to IL-27 is reduced in T cells of asthmatic patients. (A) PBMCs collected from controls and asthmatic patients were ex vivo stimulated with or without IL-27 $(10 \mathrm{ng} / \mathrm{ml})$ for the indicated duration. STAT1 and STAT3 phosphorylation was determined by flow cytometry analysis. Treg and conventional CD4 T cells were gated as CD3 ${ }^{+} C D 4+C D 25^{+} C D 127^{10 /-}$ and $C D 3^{+} C D 4^{+} C D 25-C D 127^{10 /-}$ cells, respectively. The geometric mean fluorescence intensity is normalized to those of untreated controls and shown as $\triangle$ gMFI. (B) IL-27R $\alpha$ expression on Tregs and conventional CD4 T cells was determined by flow cytometry. All the experiments were repeated more than twice. The data shown indicate the mean $\pm S D(n=4-6)$. ${ }^{*} P<0.05$, ${ }^{* *} P<0.01$, as determined by Mann-Whitney nonparametric test.

model system, that IL-27 signaling in Tregs plays an important role in limiting colitogenic Th1/Th17 type responses (28). Since this model requires a lymphopenic system to induce inflammation, we next developed Treg-specific Il27ra $\mathrm{ra}^{-1-}$ animal models and tested the role of IL-27 in Tregs during myelin antigen-reactive Th17 type autoimmune inflammation (19). Analogous to colitis models, we found that IL-27 signaling in Tregs plays an instrumental role in controlling Th1/Th17 type autoimmune inflammation. The results reported in this study demonstrate that, in addition to Th1/Th17 type inflammation, IL-27 signaling also plays an important role in Tregs to limit Th2-type allergic lung inflammation.

IL-27 intranasally administered during allergen challenge greatly diminished the development of allergic airway inflammation, and the inhibition was completely abolished in endogenous Treg-depleted mice or in germline Il27ra $\mathrm{ra}^{-1-}$ mice. This unexpected finding was further corroborated by the fact that the failure of IL-27 to inhibit allergic inflammation in these conditions was fully restored following WT Foxp $3^{+}$iTreg transfer and that IL-27 treatment, together with iTreg transfer, further improved Treg-dependent inhibition. Likewise, Treg-specific Il27ra- mice developed severe inflammatory responses, and most importantly, these mice were refractory to IL-27-mediated inhibition of inflammation.

IL-27 has several potential target cells for its immunoregulatory function in the setting of allergic airway inflammation. For example, CD4 T cell proliferation and differentiation into Th2 phenotype effector cells can directly be suppressed by IL-27 stimulation (13). Group 2 innate lymphoid cells (ILC2) produce copious amounts of $\mathrm{Th} 2$ signature cytokines and induce Th2-type inflammatory responses at the mucosal tissues such as the lung, and the ILC2 responses can also be inhibited by IL-27 signaling $(41,42)$. Given that IL-27 was unable to alter inflammatory cell infiltration in the lung tissues and T cell inflammatory cytokine productions without Tregs, it is possible that IL-27-dependent regulation of CD4 and ILC2 cell functions may be operated via Tregs.

Of note, the total numbers of lung-infiltrating Tregs were reduced after IL-27 administration. Since IL-27 antagonizes iTreg development (14), the reduction in total cell numbers may account for antigen-induced Tregs generated in vivo. Lung-infiltrating Tregs predominantly expressed the Nrp1, suggesting that they are of thymic origin (43). However, in vivo-induced Tregs may also acquire Nrp1 expression (44). 
Their precise origin during CA-induced allergic inflammation remains to be determined. We found that both thymus-derived Tregs and in vitro-generated iTregs similarly responded to IL-27 stimulation in vitro (Le HT and Min B, unpublished observation). Nevertheless, IL-27 signaling in the Foxp3 ${ }^{+}$Tregs appears to be responsible and sufficient for the suppression of lung inflammation because IL-27 is unable to diminish lung inflammation in the absence of Tregs or of Treg expression of the IL-27R $\alpha$.

What is, then, the underlying mechanism by which IL-27 controls Treg functions? Treg expression of suppressive molecules, including ICOS, CD39, and CD73, remained unchanged by the treatment, suggesting that they are not involved. We previously reported that IL-27 stimulation in Tregs induces surface expression of Lag3, a negative regulator of $\mathrm{T}$ cell activation, and that Lag3 expression of Tregs is crucial for Treg suppression in the model of intestinal inflammation (28). Lag3 was initially reported to be essential for Treg functions because $\mathrm{Lag} 3^{-/-}$Tregs display reduced suppressive activity (45). Consistent with these findings, we found that $\mathrm{Lag}^{-/-}$Tregs adoptively transferred were unable to attenuate allergic inflammation in Treg-depleted recipients even with systemic IL-27 administration, demonstrating a critical role of the IL-27/Lag3 axis in mediating Treg control of allergic inflammation. It was recently reported in the model of autoimmune diabetes that Lag3 intrinsically limits Treg proliferation and function at the sites of inflammation (46). The discrepancy behind these findings is unclear, and the precise contribution of Lag3 in Treg functions needs to be determined.

IL-27 induces IL-10-producing Foxp3- Tr1 cells, and Tr1 cells are implicated in immune tolerance $(47,48)$. Indeed, Tr1 cells suppress Th2-type cytokine production from allergen-activated effector CD4 T cells, and their numbers or functions were found to be reduced in allergic patients $(49,50)$. However, we did not find evidence that IL-27 treatment increases the generation of IL-10+ CD4 T cells in vivo. Instead, accumulation of IL-10+ CD4 T cells in the lung was rather decreased after IL-27 administration, and the reduction was dependent on Tregs. While IL-27 is a potent inducer of IL-10 expression in activated T cells in vitro $(51,52)$, whether IL-27 is equally capable of doing so in vivo is less clear. IL-27-Fc administered after collagen-induced arthritis induction ameliorates the disease severity and incidence; however, IL-10 producing cells remained unchanged by the treatment (53). Unlike an in vitro cell culture system, there are multiple cell types potentially competing for IL-27 in vivo, among which Foxp $3^{+}$Tregs may be favored targets of IL-27. While it is formally possible that the absence of Tregs would then allow other target cells to respond to IL-27, severe inflammation in Treg-specific Il27 $\mathrm{ra}^{-1-}$ mice and the inability of IL-27 to prevent the inflammation in this condition strongly suggest that IL-27 signaling in Tregs, but not in effector T cells, is the main pathway through which IL-27 mediates its function in vivo.

Immunoregulatory roles of IL-27 in the airways during respiratory infection have previously been examined. Liu et al. reported that delayed production of IL-27 limits immunopathology and dampens Th1/Th17 immune responses via IL-10-dependent and -independent pathways, while preserving defense during influenza virus infection (54). Likewise, Hunter and colleagues reported that endogenous IL-27 regulates Th2-biased immune responses during respiratory paramyxovirus infection (55). However, these reports did not identify the precise target cells of IL-27 for the protective functions. Pyle et al. demonstrated, using respiratory syncytial virus infection models, that IL-27 produced in the airways following the infection promotes local Treg functions to dampen virus-specific T cell immunity and that IL-27 is a key cytokine to induce molecules associated with Treg-suppressive function, including KLRG1 and GITR (56). These findings fit very well with the current study that Tregs are important target cells of IL-27, although Lag3 was not found to be induced by IL-27. The discrepancy may be due to the model systems used.

It was previously reported that CD4 T cells from asthmatic patients display impaired STAT1 phosphorylation in response to IL-27 stimulation (57), which is likely attributed to upregulated Socs3 expression by IL-4 stimulation. Similarly, we observed poor STAT1 phosphorylation in CD4 T cells from asthmatic patients after ex vivo IL-27 stimulation. We also found that IL-27-induced STAT1 phosphorylation in Tregs is severely impaired. IL-27 also activates STAT3 pathway, and the IL-27/STAT3 axis has previously been shown to be critical for IL-27-induced proliferation (30), PD-L1 expression (32), and inhibition of inducible Treg development (31). STAT3 phosphorylation in response to IL-27 stimulation was somewhat diminished in conventional CD4 T cells and Tregs from asthmatic patients, although the extent of reduction was less obvious than that of pSTAT1. Given that surface expression of the IL-27R was comparable between the patients and control subjects, the proximal signaling events involving STAT phosphorylation may be selectively impaired in patients. Impaired IL-27-mediated activation in Tregs of asthmatic patients may be responsible for their inadequate regulatory functions, which then perpetuate inflammatory responses. It will 
be important to explore the molecular basis underlying the defects of IL-27 signaling in different cell types of asthmatics versus control subjects. IL-27 production is increased in asthmatic patients, and there seems to be a combined effect between IL-27 with the chemokine CCL26/CXCL9 expression, which could identify a more severe asthma phenotype (58). Increased IL-27 production in severe asthma may be the secondary effect resulted from robust inflammatory reactions. It is possible that the magnitude of IL-27 production may have different effects on various target cells and further on the development of allergic inflammation.

IL-27 has previously been shown to be produced by alveolar macrophages in response to house dust mite antigen challenge (59). During respiratory virus infection, multiple myeloid cells, including monocytes, macrophages, neutrophils, and DCs, can produce IL-27 (56). However, given the facts that multiple cell types express the IL-27R $\alpha$ and that Tregs are the predominant responders of IL-27 in vivo, it is possible that Tregs may be properly positioned around the source at the site of inflammation to efficiently utilize it. We are currently examining the precise source of IL-27 during CA-induced airway inflammation.

In summary, our findings highlight a role of Tregs in mediating regulatory functions of IL-27 in downregulating allergic inflammatory responses. Despite the fact that there are multiple types of IL-27R ${ }^{+}$cells capable of responding to the cytokine, our results suggest that Treg response to IL-27 is a requisite for IL-27-induced immune regulation. Since IL-27 is considered a novel therapeutic tool for inflammatory disorders $(60,61)$, improving (or restoring) IL-27 responsiveness of Tregs may be an important goal to achieve in future investigations.

\section{Methods}

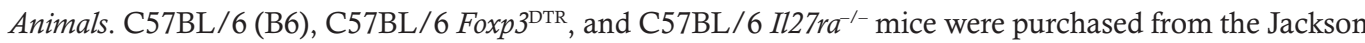
Laboratory. C57BL/6 Foxp3 ${ }^{\mathrm{GFP}}$ (62) and C57BL/6 Lag3 $^{-1-}$ (63) mice were obtained from Yasmine Belkaid (NIAID, NIH) and Christoph Benoist (Harvard Medical School, Boston, Massachusetts, USA), respectively. Treg-specific Il27 $\mathrm{ra}^{-/-}$(Treg ${ }^{\Delta I l 27 r a}$ ) mice were previously reported (19). All of the mice were maintained in a specific pathogen-free facility located in the Lerner Research Institute.

Human studies. PBMC were collected from 6 asthmatic patients (4 severe and 2 nonsevere asthmatic) and 6 controls. Age and race was not different between the 2 groups [Age (yrs) mean \pm SD: controls, $41.2 \pm$ 8.8; Asthmatics, $47.5 \pm 15.7 ; \mathrm{p}=0.42$; Race, African American/Caucasian: controls, 1/5; Asthmatics 3/3; $\mathrm{p}=0.22$ ]. However, sex was significantly different [Sex, M/F: controls, 4/2; asthmatics, 0/6; $p=0.0143$ ]. Asthma was confirmed based on responsiveness to $\beta$-agonist $(\geq 12 \%$ increase in forced expired volume in 1 second $\left[\mathrm{FEV}_{1}\right.$ from baseline post-albuterol] or (if $<12 \%$ ), a positive methacholine bronchoprovocation. Severe asthma was defined according to a modification of the European Respiratory Society/American Thoracic Society consensus definition (64). Control subjects lacked cardiopulmonary symptoms and had normal spirometry and negative methacholine challenge. Exclusion criteria for both asthmatics and controls included age less than 18 years, pregnancy, current smoking, smoking within the past year, or former smokers with $\geq 5$ pack-year total history. All participants signed an informed consent, adherent to the Declaration of Helsinki and approved by the Cleveland Clinic Review Board.

Airway inflammation. Mice were injected i.p. on days 0 and 7 with $5 \mathrm{mg}$ of CA (Greer Laboratory) mixed in $100 \mu 1$ of alum adjuvant (aluminum hydroxide; MilliporeSigma). Starting on day 14 , the mice were intranasally challenged for 4 consecutive days with CA alone or together with 100 ng recombinant mouse IL-27 (rIL-27; 2799-ML/CF, R\&D Systems). Mice were sacrificed 24 hours after the last Ag challenge. In some experiments, Tregs were depleted by i.p. injection of $1 \mathrm{mg}$ of DTX (MilliporeSigma). Lung tissues were prepared from paraffin-embedded blocks and stained with H\&E. Inflammation was scored as previously reported (20). Lung mechanics was measured using the FlexiVent ventilator (FlexiVent, Scireq). Lung resistance was measured in response to increasing doses of inhaled methacholine, as previously described (65).

Flow cytometry. At sacrifice, BALF cells, lung tissue, and draining medLN were collected and prepared as previously described (20). Briefly, the cells were stained with anti-CD4 (RM4-5, BioLegend), anti-Ly6G (1A8, BD Biosciences), anti-Siglec-F (1RNM44N, eBioscience), anti-ICOS (C398.4A, BD Biosciences), anti-Nrp1 (3E12, BioLegend), anti-CD39 (24DMS1, eBioscience), anti-CD73 (TY/23, BD Biosciences), and anti-BrdU (3D4, BD Biosciences). For intracellular staining, cells were stained with anti-IL-4 (11B11, eBioscience), anti-IL-13 (eBio13A, eBioscience), anti-IL-17 (TC11-18H10, BD Bioscience), anti-IFN- $\gamma$ (XMG1.2, BD Bioscience), anti-IL-5 (TRFK5, BD Biosciences), anti-IL-10 (JES5-16E3, BD Biosciences) and anti-IL-2 (JES6-5H4, BD Biosciences). Cells were acquired using a FACSLSR II (BD Biosciences) and analyzed using a FlowJo software (Tree Star Inc.). For intracellular staining, cells were harvested separately 
and stimulated ex vivo with PMA (10 ng/ml, MilliporeSigma) and ionomycin ( $1 \mu \mathrm{M}$, MilliporeSigma) for 4 hours in the presence of $2 \mu \mathrm{M}$ monensin (Calbiochem) during the last 2 hours of stimulation. Cells were immediately fixed with $4 \%$ paraformaldehyde, permeabilized, and stained with fluorescence-conjugated Abs. In some experiments, mice were injected with $1 \mathrm{mg}$ of BrdU (MilliporeSigma) 24 hours prior to sacrifice. In vivo BrdU incorporation was measured using a BrdU kit (BD Pharmingen), according to the manufacturer's instructions.

PBMCs from asthmatic patients $(n=6)$ and control donors $(n=6)$ were isolated using Isoprep. To detect pSTAT1 and pSTAT3, PBMCs were stimulated with or without recombinant human IL-27 (rhIL-27) $(10 \mathrm{ng} / \mathrm{ml})$ for the indicated duration. STAT1 and STAT3 phosphorylation was determined by flow cytometry analysis. Treg and conventional CD4 T cells were gated as $\mathrm{CD}^{+} \mathrm{CD} 4{ }^{+} \mathrm{CD} 25^{+} \mathrm{CD} 127^{-}$and $\mathrm{CD}^{+}{ }^{+} \mathrm{CD} 4{ }^{+} \mathrm{CD} 25^{-} \mathrm{CD} 127^{+}$cells, respectively.

In vitro generation of $i$ Treg. Naive $\mathrm{CD}^{+}{ }^{+} \mathrm{Foxp}^{-} \mathrm{CD} 44^{\text {lo }} \mathrm{T}$ cells from lymph nodes of Foxp $3^{\mathrm{GFP}}$ mice were sorted by cell sorting and treated with plate-bound anti-CD3 mAb (clone 2C11), soluble anti-CD28 mAb (clone 37.51), and $100 \mathrm{U} / \mathrm{ml}$ rhIL-2 (obtained from the Blood Resources Branch, NCI) in the presence of TGF- $\beta$ for 3 days. iTreg cells (CD4 Foxp $3^{+}$) cells were sorted by flow cytometry prior to transfer.

Cytokine analysis by a cytometric bead array. In some experiments, BALF was collected from mice induced for allergic airway inflammation and treated with rIL-27, PBS, DTX, or adoptive Foxp3 ${ }^{+}$Treg transfer. IL-4 and IL-13 secretion was quantified using a cytometric bead array (CBA) kit (BD Biosciences) and an enhanced sensitivity flex set (BD Biosciences), respectively, according to the manufacturer's instructions.

Gene expression analysis. RNA was isolated from inflamed lung tissues or FACS cells using a GeneJet RNA isolation kit (Thermo Fisher Scientific). cDNA was synthesized using Moloney Murine Leukemia Virus (M-MLV) reverse transcriptase (Promega). Real-time PCR analysis was performed using an ABI7500 real-time PCR system (Applied Biosystem) and gene specific Taqman primers.

Statistics. Statistical significance was determined by the Mann-Whitney (2-tailed) or Kruskal-Wallis test using the Prism 7 software (GraphPad). $P<0.05$ was considered statistically significant.

Study approval. All animal experiments were performed in accordance with protocols approved by the Cleveland Clinic Foundation IACUC (animal protocol no. 2016-1705). All human studies were approved by the Cleveland Clinic IRB (IRB no. PPG8351). All subjects were recruited from Cleveland Clinic and gave written informed consent by signing a consent document approved by the Cleveland Clinic IRB.

\section{Author contributions}

QTN performed most of the experiments, analyzed the data, and wrote the manuscript. EJ, HTL, SK, DK, $\mathrm{ND}$, and KA helped with experiments and analyzed data. MAA, WMB, and SC helped with the setup and analysis of the data. BM designed the experiments, analyzed the data, and wrote the manuscript.

\section{Acknowledgments}

The authors thank Serpil Erzurum for critical review of the manuscript and Kelly Weiss for technical assistance. This work was supported by grants from the American Asthma Foundation and NIH (R21-AI121524, R01-AI125247, P01-HL081064).

Address correspondence to: Booki Min, Department of Inflammation and Immunity/NB30, Lerner Research Institute, Cleveland Clinic Foundation, 9500 Euclid Ave, Cleveland, Ohio 44195, USA. Phone: 216.445.3126; Email: minb@ccf.org.

EJ's present address is: Ulsan National Institute of Science and Technology, Ulsan, South Korea.

1. Wills-Karp M. Immunologic basis of antigen-induced airway hyperresponsiveness. Annu Rev Immunol. 1999;17:255-281.

2. Lu L, Barbi J, Pan F. The regulation of immune tolerance by FOXP3. Nat Rev Immunol. 2017;17(11):703-717.

3. Miyara M, Wing K, Sakaguchi S. Therapeutic approaches to allergy and autoimmunity based on FoxP3+ regulatory T-cell activation and expansion. J Allergy Clin Immunol. 2009;123(4):749-755.

4. Rudensky AY. Regulatory T cells and Foxp3. Immunol Rev. 2011;241(1):260-268.

5. Lin W, et al. Allergic dysregulation and hyperimmunoglobulinemia E in Foxp3 mutant mice. J Allergy Clin Immunol. 2005;116(5):1106-1115.

6. Sakaguchi S, Yamaguchi T, Nomura T, Ono M. Regulatory T cells and immune tolerance. Cell. 2008;133(5):775-787.

7. Lewkowich IP, et al. CD4+CD25+ T cells protect against experimentally induced asthma and alter pulmonary dendritic cell 
phenotype and function. J Exp Med. 2005;202(11):1549-1561.

8. Kearley J, Barker JE, Robinson DS, Lloyd CM. Resolution of airway inflammation and hyperreactivity after in vivo transfer of CD4+CD25+ regulatory T cells is interleukin 10 dependent. J Exp Med. 2005;202(11):1539-1547.

9. Lin YL, Shieh CC, Wang JY. The functional insufficiency of human CD4+CD25 high T-regulatory cells in allergic asthma is subjected to TNF-alpha modulation. Allergy. 2008;63(1):67-74.

10. Hartl D, et al. Quantitative and functional impairment of pulmonary CD4+CD25hi regulatory T cells in pediatric asthma. $J$ Allergy Clin Immunol. 2007;119(5):1258-1266.

11. Hall AO, Silver JS, Hunter CA. The immunobiology of IL-27. Adv Immunol. 2012;115:1-44.

12. Hunter CA, Kastelein R. Interleukin-27: balancing protective and pathological immunity. Immunity. 2012;37(6):960-969.

13. Artis D, et al. The IL-27 receptor (WSX-1) is an inhibitor of innate and adaptive elements of type 2 immunity. J Immunol. 2004;173(9):5626-5634.

14. Batten $\mathrm{M}$, et al. Interleukin 27 limits autoimmune encephalomyelitis by suppressing the development of interleukin 17-producing T cells. Nat Immunol. 2006;7(9):929-936.

15. Stumhofer JS, et al. Interleukin 27 negatively regulates the development of interleukin 17-producing T helper cells during chronic inflammation of the central nervous system. Nat Immunol. 2006;7(9):937-945.

16. Miyazaki Y, et al. Exacerbation of experimental allergic asthma by augmented Th2 responses in WSX-1-deficient mice. J Immunol. 2005;175(4):2401-2407.

17. Diveu C, et al. IL-27 blocks RORc expression to inhibit lineage commitment of Th17 cells. J Immunol. 2009;182(9):5748-5756.

18. Hall AO, et al. The cytokines interleukin 27 and interferon- $\gamma$ promote distinct Treg cell populations required to limit infection-induced pathology. Immunity. 2012;37(3):511-523.

19. Do J, et al. Treg-specific IL-27R $\alpha$ deletion uncovers a key role for IL-27 in Treg function to control autoimmunity. Proc Natl Acad Sci USA. 2017;114(38):10190-10195

20. Jang E, et al. Lung-Infiltrating Foxp3+ Regulatory T Cells Are Quantitatively and Qualitatively Different during Eosinophilic and Neutrophilic Allergic Airway Inflammation but Essential To Control the Inflammation. J Immunol. 2017;199(12):3943-3951.

21. Grünig G, et al. Requirement for IL-13 independently of IL-4 in experimental asthma. Science. 1998;282(5397):2261-2263.

22. Wills-Karp M, et al. Interleukin-13: central mediator of allergic asthma. Science. 1998;282(5397):2258-2261.

23. Kim JM, Rasmussen JP, Rudensky AY. Regulatory T cells prevent catastrophic autoimmunity throughout the lifespan of mice Nat Immunol. 2007;8(2):191-197.

24. Yoshida H, Hunter CA. The immunobiology of interleukin-27. Annu Rev Immunol. 2015;33:417-443.

25. Trandem K, Anghelina D, Zhao J, Perlman S. Regulatory T cells inhibit T cell proliferation and decrease demyelination in mice chronically infected with a coronavirus. J Immunol. 2010;184(8):4391-4400.

26. Shevach EM. Mechanisms of foxp3+ T regulatory cell-mediated suppression. Immunity. 2009;30(5):636-645.

27. Sakaguchi S, Wing K, Onishi Y, Prieto-Martin P, Yamaguchi T. Regulatory T cells: how do they suppress immune responses? Int Immunol. 2009;21(10):1105-1111.

28. Do JS, et al. An IL-27/Lag3 axis enhances Foxp3+ regulatory T cell-suppressive function and therapeutic efficacy. Mucosal Immunol. 2016;9(1):137-145.

29. Rubtsov YP, et al. Regulatory T cell-derived interleukin-10 limits inflammation at environmental interfaces. Immunity. 2008;28(4):546-558.

30. Owaki T, et al. STAT3 is indispensable to IL-27-mediated cell proliferation but not to IL-27-induced Th1 differentiation and suppression of proinflammatory cytokine production. J Immunol. 2008;180(5):2903-2911.

31. Huber M, et al. IL-27 inhibits the development of regulatory T cells via STAT3. Int Immunol. 2008;20(2):223-234

32. Horlad H, et al. An IL-27/Stat3 axis induces expression of programmed cell death 1 ligands (PD-L1/2) on infiltrating macrophages in lymphoma. Cancer Sci. 2016;107(11):1696-1704.

33. Kamiya S, Owaki T, Morishima N, Fukai F, Mizuguchi J, Yoshimoto T. An indispensable role for STAT1 in IL-27-induced T-bet expression but not proliferation of naive CD4+ T cells. J Immunol. 2004;173(6):3871-3877.

34. Takeda A, et al. Cutting edge: role of IL-27/WSX-1 signaling for induction of T-bet through activation of STAT1 during initial Th1 commitment. J Immunol. 2003;170(10):4886-4890.

35. Zhang S, et al. High susceptibility to liver injury in IL-27 p28 conditional knockout mice involves intrinsic interferon- $\gamma$ dysregulation of CD4+ T cells. Hepatology. 2013;57(4):1620-1631.

36. Fujimoto H, et al. IL-27 inhibits hyperglycemia and pancreatic islet inflammation induced by streptozotocin in mice. Am J Pathol. 2011;179(5):2327-2336.

37. Sweeney CM, et al. IL-27 mediates the response to IFN- $\beta$ therapy in multiple sclerosis patients by inhibiting Th17 cells. Brain Behav Immun. 2011;25(6):1170-1181.

38. Su X, et al. IL-27 attenuates airway inflammation in a mouse asthma model via the STAT1 and GADD45 $\gamma / \mathrm{p} 38 \mathrm{MAPK}$ pathways. J Transl Med. 2016;14(1):283.

39. Yoshimoto T, Yoshimoto T, Yasuda K, Mizuguchi J, Nakanishi K. IL-27 suppresses Th2 cell development and Th2 cytokines production from polarized Th2 cells: a novel therapeutic way for Th2-mediated allergic inflammation. J Immunol. 2007;179(7):4415-4423.

40. Fujita H, et al. Production of both IL-27 and IFN-gamma after the treatment with a ligand for invariant NK T cells is responsible for the suppression of Th2 response and allergic inflammation in a mouse experimental asthma model. J Immunol. 2009;183(1):254-260.

41. Mchedlidze T, Kindermann M, Neves AT, Voehringer D, Neurath MF, Wirtz S. IL-27 suppresses type 2 immune responses in vivo via direct effects on group 2 innate lymphoid cells. Mucosal Immunol. 2016;9(6):1384-1394.

42. Moro K, et al. Interferon and IL-27 antagonize the function of group 2 innate lymphoid cells and type 2 innate immune responses. Nat Immunol. 2016;17(1):76-86.

43. Yadav M, et al. Neuropilin-1 distinguishes natural and inducible regulatory T cells among regulatory T cell subsets in vivo. $J$ Exp Med. 2012;209(10):1713-1722. 
44. Chen TT. Statistical issues and challenges in immuno-oncology. J Immunother Cancer. 2013;1:18.

45. Huang CT, et al. Role of LAG-3 in regulatory T cells. Immunity. 2004;21(4):503-513.

46. Zhang Q, et al. LAG3 limits regulatory T cell proliferation and function in autoimmune diabetes. Sci Immunol. 2017;2(9):eaah4569.

47. Pot C, Apetoh L, Kuchroo VK. Type 1 regulatory T cells (Tr1) in autoimmunity. Semin Immunol. 2011;23(3):202-208.

48. Akdis CA, Akdis M. Mechanisms of immune tolerance to allergens: role of IL-10 and Tregs. J Clin Invest. 2014;124(11):4678-4680.

49. Akdis M, et al. Immune responses in healthy and allergic individuals are characterized by a fine balance between allergen-specific T regulatory 1 and T helper 2 cells. J Exp Med. 2004;199(11):1567-1575.

50. Pellerin L, et al. Peanut-specific type 1 regulatory T cells induced in vitro from allergic subjects are functionally impaired. $J$ Allergy Clin Immunol. 2018;141(1):202-213.e8.

51. Batten M, Kljavin NM, Li J, Walter MJ, de Sauvage FJ, Ghilardi N. Cutting edge: IL-27 is a potent inducer of IL-10 but not FoxP3 in murine T cells. J Immunol. 2008;180(5):2752-2756.

52. Murugaiyan G, Mittal A, Lopez-Diego R, Maier LM, Anderson DE, Weiner HL. IL-27 is a key regulator of IL-10 and IL-17 production by human CD4+ T cells. J Immunol. 2009;183(4):2435-2443.

53. Moon SJ, et al. In vivo action of IL-27: reciprocal regulation of Th17 and Treg cells in collagen-induced arthritis. Exp Mol Med. 2013;45:e46.

54. Liu FD, et al. Timed action of IL-27 protects from immunopathology while preserving defense in influenza. PLoS Pathog. 2014;10(5):e1004110.

55. Muallem G, et al. IL-27 Limits Type 2 Immunopathology Following Parainfluenza Virus Infection. PLoS Pathog. 2017;13(1):e1006173.

56. Pyle CJ, Uwadiae FI, Swieboda DP, Harker JA. Early IL-6 signalling promotes IL-27 dependent maturation of regulatory T cells in the lungs and resolution of viral immunopathology. PLoS Pathog. 2017;13(9):e1006640.

57. Chen Z, et al. IL-4 confers resistance to IL-27-mediated suppression on CD4+ T cells by impairing signal transducer and activator of transcription 1 signaling. J Allergy Clin Immunol. 2013;132(4):912-21.e1.

58. Xie M, et al. IL-27 and type 2 immunity in asthmatic patients: association with severity, CXCL9, and signal transducer and activator of transcription signaling. J Allergy Clin Immunol. 2015;135(2):386-394.

59. Mathie SA, et al. Alveolar macrophages are sentinels of murine pulmonary homeostasis following inhaled antigen challenge. Allergy. 2015;70(1):80-89.

60. Andrews C, McLean MH, Durum SK. Interleukin-27 as a Novel Therapy for Inflammatory Bowel Disease: A Critical Review of the Literature. Inflamm Bowel Dis. 2016;22(9):2255-2264.

61. Meka RR, Venkatesha SH, Dudics S, Acharya B, Moudgil KD. IL-27-induced modulation of autoimmunity and its therapeutic potential. Autoimmun Rev. 2015;14(12):1131-1141.

62. Bettelli E, et al. Reciprocal developmental pathways for the generation of pathogenic effector TH17 and regulatory T cells Nature. 2006;441(7090):235-238.

63. Miyazaki T, Dierich A, Benoist C, Mathis D. Independent modes of natural killing distinguished in mice lacking Lag3. Science. 1996;272(5260):405-408.

64. Chung KF, et al. International ERS/ATS guidelines on definition, evaluation and treatment of severe asthma. Eur Respir J. 2014;43(2):343-373.

65. Asosingh K, et al. Nascent endothelium initiates Th2 polarization of asthma. J Immunol. 2013;190(7):3458-3465. 